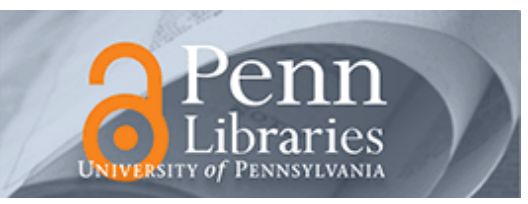

University of Pennsylvania

ScholarlyCommons

August 2002

\title{
Synchronization Recovery of Chaotic Wave Through an Imperfect Channel
}

\author{
Xiaomin Yang \\ University of Central Florida \\ Thomas X. Wu \\ University of Central Florida \\ Dwight L. Jaggard \\ University of Pennsylvania, jaggard@seas.upenn.edu
}

Follow this and additional works at: https://repository.upenn.edu/ese_papers

\section{Recommended Citation}

Xiaomin Yang, Thomas X. Wu, and Dwight L. Jaggard, "Synchronization Recovery of Chaotic Wave Through an Imperfect Channel", . August 2002.

Copyright 2002 IEEE. Reprinted from IEEE Antennas and Wireless Propagation Letters, Volume 1, Issue 8, August 2002, pages 154-156.

Publisher URL: http://ieeexplore.ieee.org/xpl/tocresult.jsp?isNumber=26317\&puNumber=7727

This material is posted here with permission of the IEEE. Such permission of the IEEE does not in any way imply IEEE endorsement of any of the University of Pennsylvania's products or services. Internal or personal use of this material is permitted. However, permission to reprint/republish this material for advertising or promotional purposes or for creating new collective works for resale or redistribution must be obtained from the IEEE by writing to pubs-permissions@ieee.org. By choosing to view this document, you agree to all provisions of the copyright laws protecting it.

This paper is posted at ScholarlyCommons. https://repository.upenn.edu/ese_papers/30

For more information, please contact repository@pobox.upenn.edu. 


\title{
Synchronization Recovery of Chaotic Wave Through an Imperfect Channel
}

\begin{abstract}
We present a novel idea to recover synchronization using the genetic algorithm after a chaotic wave passes through an imperfect channel with constant attenuation and offset. The compensation block, which is added before the receiver, is used to compensate the distortion of the imperfect channel. A new concept, the synchronization mismatch is defined and used as the cost function in genetic algorithm to design the compensation block. The validity of this approach is suggested by numerical simulations.
\end{abstract}

\section{Keywords}

Chaos, synchronization, wave propagation

\section{Comments}

Copyright 2002 IEEE. Reprinted from IEEE Antennas and Wireless Propagation Letters, Volume 1, Issue 8, August 2002, pages 154-156.

Publisher URL: http://ieeexplore.iee.. rg/xpl/tocresult.jsp?isNumber=26317\&puNumber=7727

This material is posted here with permission of the IEEE. Such permission of the IEEE does not in any way imply IEEE endorsement of any of the University of Pennsylvania's products or services. Internal or personal use of this material is permitted. However, permission to reprint/republish this material for advertising or promotional purposes or for creating new collective works for resale or redistribution must be obtained from the IEEE by writing to pubs-permissions@ieee.org. By choosing to view this document, you agree to all provisions of the copyright laws protecting it. 


\title{
Synchronization Recovery of Chaotic Wave Through an Imperfect Channel
}

\author{
Xiaomin Yang, Student Member, IEEE, Thomas X. Wu, Senior Member, IEEE, and Dwight L. Jaggard, Fellow, IEEE
}

\begin{abstract}
We present a novel idea to recover synchronization using the genetic algorithm after a chaotic wave passes through an imperfect channel with constant attenuation and offset. The compensation block, which is added before the receiver, is used to compensate the distortion of the imperfect channel. A new concept, the synchronization mismatch is defined and used as the cost function in genetic algorithm to design the compensation block. The validity of this approach is suggested by numerical simulations.
\end{abstract}

Index Terms-Chaos, synchronization, wave propagation.

\section{INTRODUCTION}

$\mathbf{T}$ HE presence of chaotic phenomena in physical and electrical systems is common and has been extensively demonstrated [1]. Chaos in physical systems was originally thought to be a form of noise or random disturbance. It is now well-known that chaos is not noise but rather an intricate and often repetitive pattern that arises in the behavior of some nonlinear systems that are extremely sensitive to changes in initial condition. Because of this sensitivity, the application of chaos to system design was originally thought to be difficult. However, it has been demonstrated that certain chaotic systems possess the property of synchronization and can be subject to control [2]. This has piqued interest in new, nonlinear devices and systems for secure communications [3]-[7] and other applications. Communication systems, especially wireless systems, based on the superior performance of nonlinear technology operating in the chaotic regime are expected to play an increasing role in commercial and other communications systems.

Chaotic communication systems, both digital and analog, require high-frequency circuits and systems for transmission and detection. The use of synchronized chaotic systems for communications usually relies on the robustness of the synchronization within the transmitter-receiver pair. Recently, Wu and Jaggard [8], [9] investigated chaotic wave propagation through an imperfect channel. A new concept, synchronization mismatch, was introduced and it was found that if the communication channel was imperfect, the distorted signal at the receiver input might cause a considerable synchronization mismatch between the transmitter and receiver systems.

The genetic algorithm is a subset of evolutionary algorithms that approximate some biological growth processes to optimize

Manuscript received August 20, 2002.

$\mathrm{X}$. Yang and T. X. Wu are with the School of Electrical Engineering and Computer Science, University of Central Florida, Orlando, FL 32816-2450 USA (tomwu@mail.ucf.edu).

D. L. Jaggard is with the Complex Media Laboratory, Department of Electrical and Systems Engineering, University of Pennsylvania, Philadelphia, PA 19104-6390 USA.

Digital Object Identifier 10.1109/LAWP.2002.807569 highly complex cost functions. The genetic algorithm allows a population composed of many individuals to evolve under specified selection rules to a state that maximizes the "fitness" (i.e., minimizes the cost function) [10]. It has been used in many applications in communication systems, such as communication network [11], [12], multi-user detection [13], wide-band antenna design [14], and communication controller design [15].

In this paper, we propose a novel approach using genetic algorithm to recover synchronization after a chaotic wave propagates through an imperfect channel with constant attenuation and offset. To achieve this result, a compensator is added before the receiver. The genetic algorithm is applied to estimate the attenuation and offset. From the examples given here, we find that the chaotic synchronization may be recovered efficiently. Although the results are based on the Lorenz systems, they can be extended to other systems as well.

\section{ChAOTIC SyNChRONIZATION}

The concept of chaotic synchronization in a communication system is shown schematically in Fig. 1. Here, we use the Lorenz systems as examples and assume that the transmitter and receiver are identical Lorenz systems, except that for the receiver system we use the receiver input signal $\tilde{u}_{1}$ to replace the receiver output signal $u_{2}$ in some of the expressions. The dynamic equations for the two systems are, respectively,

$$
\begin{gathered}
\left\{\begin{array} { l } 
{ u _ { 1 } ^ { \prime } = K \alpha ( v _ { 1 } - u _ { 1 } ) } \\
{ v _ { 1 } ^ { \prime } = K ( \rho u _ { 1 } - v _ { 1 } - u _ { 1 } w _ { 1 } ) } \\
{ w _ { 1 } ^ { \prime } = K ( u _ { 1 } v _ { 1 } - \beta w _ { 1 } ) }
\end{array} \text { and } \left\{\begin{array}{l}
u_{2}^{\prime}=K \alpha\left(v_{2}-u_{2}\right) \\
v_{2}^{\prime}=K\left(\rho \tilde{u}_{1}-v_{2}-\tilde{u}_{1} w_{2}\right) \\
w_{2}^{\prime}=K\left(\tilde{u}_{1} v_{2}-\beta w_{2}\right) .
\end{array}\right.\right.
\end{gathered}
$$

Here, the prime ' denotes derivative with respect to time $t$, and $K$ is a scaling factor. It is noted that we use $\tilde{u}_{1}$ as input signal (drive signal) instead of $u_{2}$ on the right-hand side of the last two equations of the response system.

If the input signal $\tilde{u}_{1}$ of the response system is the same as $u_{1}$ of the drive system (i.e., the channel is perfect), these two systems will synchronize. This means that if these two systems start from different initial conditions, but share the same set of parameters $\rho, \alpha, \beta$, and $K$, the variables $u_{2}, v_{2}$ and $w_{2}$ of the response system will soon approach the values $u_{1}, v_{1}$ and $w_{1}$, respectively, of the drive system [16]. Likewise, if we choose $\tilde{u}_{1}$ to be a delayed version of $u_{1}$ (the channel is a time delayed system), $u_{2}, v_{2}$ and $w_{2}$ will also soon approach $u_{1}, v_{1}$ and $w_{1}$, respectively. 


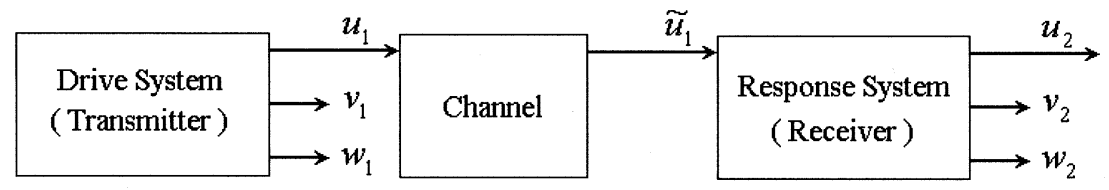

Fig. 1. A chaotic synchronization system. We assume that the transmitter and receiver are Lorenz systems sharing the same set of parameters, so that the outputs of the two systems are synchronized.

However, if $\tilde{u}_{1}$ is a distorted version of $u_{1}$ after passing through a channel, there will be a synchronization mismatch [4] between $u_{1}$ and $u_{2}$. We can quantitatively define the synchronization mismatch $(S M)$ as a dimensionless quantity: the maximum absolute value of the difference between $\tilde{u}_{1}$ and $u_{2}$ over the root mean square (rms) value of $u_{1}$, which is

$$
S M=\frac{\max _{t_{c} \leq t \leq \infty}\left|u_{2}(t)-\tilde{u}_{1}(t)\right|}{u_{\mathrm{rms}}}
$$

where $t_{c}$ can be chosen to be a time value after which the difference between $\tilde{u}_{1}$ and $u_{2}$ becomes less significant for a perfect channel. $u_{\text {rms }}$ is the rms value of $u_{1}$ and defined as

$$
u_{\mathrm{rms}}=\lim _{t \rightarrow \infty} \frac{1}{t-t_{c}} \sqrt{\int_{t_{c}}^{t}\left|u_{1}(t)\right|^{2} d t} .
$$

The synchronization mismatch defined above may be considered as a signature of the channel.

For a perfect channel, $S M$ is very small. However, for an imperfect channel, the distortion from the channel may introduce a considerable $S M$, or even destroy the chaotic synchronization completely.

\section{SYNCHRONIZATION RECOVERY AND COMPENSATOR DESIGN}

\section{A. Imperfect Channel With Attenuation and Offset}

If the channel is imperfect, the synchronization mismatch may be quite large. Let us consider an imperfect channel with attenuation and offset. In Fig. 1, the output $\hat{u}_{1}$ of the channel is given by $\hat{u}_{1}=u_{1} / A+B$, where $A$ is the attenuation factor and $B$ is the offset of the channel. The values of $A$ and $B$ will affect the synchronization status. We can find that when $A$ is as small as 1.1, the synchronization mismatch can be detected, although it is not significant. However, if $A$ goes up to 5, the mismatch can be considerable. The offset $B$ has the similar effect on the synchronization as the attenuation factor $A$. When offset $B$ increases from 1 to 5 , the mismatch grows up simultaneously. As shown in Fig. 2, when the distortion from the channel is characterized as $A=1.5$ and $B=5$, the synchronization mismatch is very significant and the synchronization can hardly hold. So far it is very clear that the mismatch increases as the attenuation or offset increases. The even worse case is that the synchronization may be totally lost.

\section{B. Synchronization Recovery}

In the following, we will show the idea to recover the chaotic synchronization. An intuitive way is to add a compensation

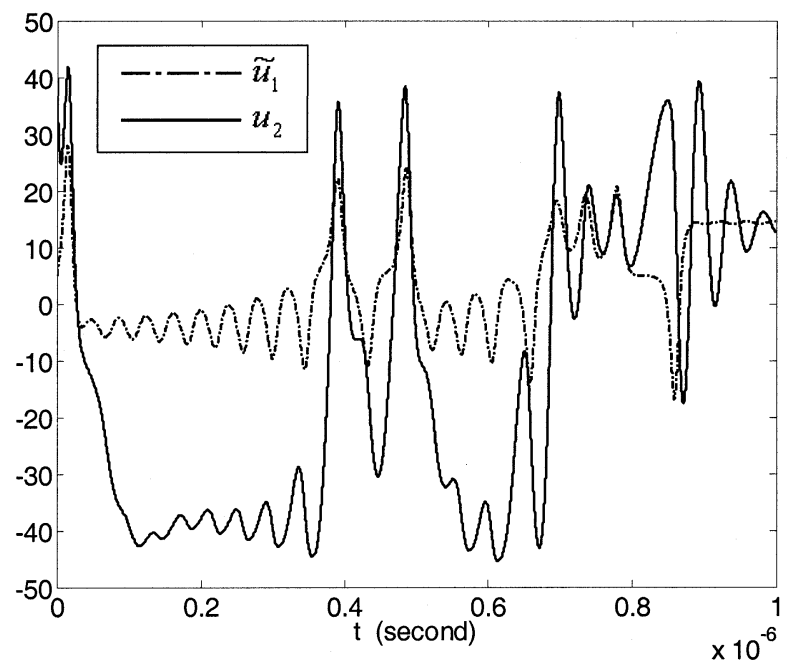

Fig. 2. Due to the distortion from the imperfect channel, attenuation factor $A=1.5$ and offset $B=5$, the chaotic synchronization between the receiver input $\tilde{u}_{1}$ (dashed line) and the receiver output $u_{2}$ (solid line) cannot hold. The Lorenz system parameters are $\alpha=16, \beta=4, \rho=50$ and $K=10^{7}$.

system before the receiver as shown in Fig. 3. We can design the compensator to be

$$
\tilde{u}_{1}=\left(\hat{u}_{1}-\hat{B}\right) \cdot \hat{A} .
$$

Therefore,

$$
\tilde{u}_{1}=\left(\hat{u}_{1}-\hat{B}\right) \cdot \hat{A}=\frac{u_{1}}{A} \cdot \hat{A}+(B-\hat{B}) \cdot \hat{A} .
$$

From (6), we can find that if $\hat{A}$ and $\hat{B}$ approach $A$ and $B$, respectively, $\tilde{u}_{1}$ will approach $u_{1}$, and the synchronization can be recovered by the response system.

Here, the objective is to find the estimation values of $\hat{A}$ and $\hat{B}$. In this paper, we use the genetic algorithm to obtain these values. The cost function used in optimization is the synchronization mismatch between the receiver input and receiver output. $\hat{A}$ and $\hat{B}$ are the approximations of $A$ and $B$ when the value of the cost function approaches zero.

\section{Numerical Example}

Let us consider an example to show the robustness of our approach to recover synchronization. For the systems in (1) and (2), the Lorenz system parameters are $\alpha=16$, and $\beta=4, \rho=50$ and $K=10^{7}$. The distortion from the channel is characterized as $A=1.5$ and $B=5$. As shown in Fig. 2, the synchronization mismatch is very significant and the synchronization can hardly hold.

Using genetic algorithm, the initial guesses are in the range $[0,5]$ for $\hat{A}$ and $[-10,10]$ for $\hat{B}$ As shown in Fig. 4, the value of 


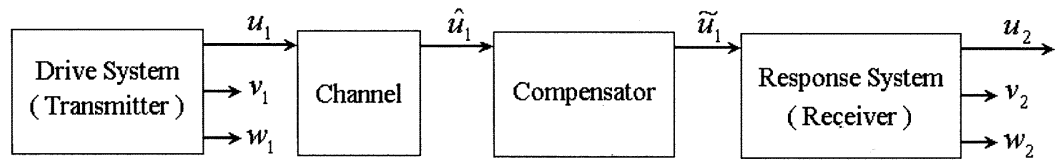

Fig. 3. A chaotic synchronization system with a compensator. We assume that the transmitter and receiver are Lorenz systems sharing the same set of parameters.
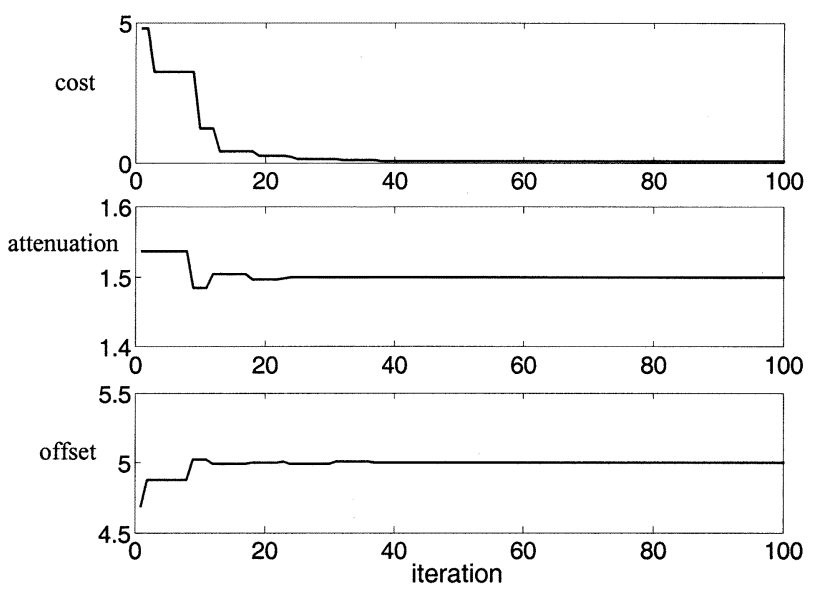

Fig. 4. Parameters of the compensation system found by genetic algorithm. An intuitive way to recover the chaotic synchronization is to add a compensator before the receiver (Fig. 3). (a) Cost versus iteration. (b) Attenuation versus iteration. (c) Offset versus iteration.



Fig. 5. The recovery of chaotic synchronization between the receiver input $\hat{u}_{1}$ (dashed line) and the receiver output $u_{2}$ (solid line). The Lorenz system parameters are $\alpha=16, \beta=4, \rho=50$ and $K=10^{7}$.

the cost function approaches zero gradually. At the meanwhile, $\hat{A}$ and $\hat{B}$ are also varying toward their optimum values. After 100 iterations, the results are: $\hat{A}=1.4996$ and $\hat{B}=5.0027$. The results are very close to the actual values of $A$ and $B$, where $A=1.5$ and $B=5$. Applying $\hat{A}$ and $\hat{B}$ in (4), the expression of the compensator becomes

$$
\tilde{u}_{1}=\left(\hat{u}_{1}-\hat{B}\right) \cdot \hat{A}=\left(\hat{u}_{1}-5.0027\right) \cdot(1.4996) .
$$

And, as shown in Fig. 5, with the compensator in (7) added before the receiver, the synchronization mismatch has been reduced remarkably. The synchronization is excellently recovered by the compensator.

\section{CONCLUSION}

In this paper, we have proposed a novel idea to use the genetic algorithm to recover synchronization after a chaotic wave passing through an imperfect channel with constant attenuation and offset. The simulation results have shown the robustness of our approach. Although this paper is based on the Lorenz systems, it can be extended to other systems as well.

\section{REFERENCES}

[1] E. Ott, Chaos in Dynamical Systems. New York: Cambridge Univ. Press, 1992.

[2] L. M. Pecora and T. L. Carroll, "Synchronization in chaotic systems," Phys. Rev. Lett., vol. 64, pp. 821-824, 1990.

[3] K. M. Cuomo et al., "Synchronization of Lorenz-based chaotic circuits with applications to communications," IEEE Trans. Circuits Syst. II, vol. 40, pp. 626-633, Oct. 1993.

[4] T. Liao and N. Huang, "An observer-based approach for chaotic synchronization with applications to secure communications," IEEE Trans. Circuits Syst.—I: Fundamental Theory Applicat., vol. 46, pp. 1144-1150, Sept. 1999.

[5] Z. Jiang, "A note on chaotic secure communication systems," IEEE Trans. Circuits Syst.—I: Fundamental Theory Applicat., vol. 49, pp. 92-96, Jan. 2002.

[6] T. Yang and L. Chua, "Secure communication via chaotic parameter modulation," IEEE Trans. Circuits Syst.-I: Fundamental Theory Applicat., vol. 43, pp. 817-819, Sept. 1996.

[7] G. Kolumban, M. P. Kennedy, and L. O. Chua, "The role of synchronization in digital communications using chaos-Part II: Chaotic modulation and chaotic synchronization," IEEE Trans. Circuits Syst.-I: Fundamental Theory Applicat., vol. 45, pp. 1129-1140, Nov. 1998.

[8] T. X. Wu and D. L. Jaggard, "Chaotic wave scattering and synchronization," in Proc. IEEE Int. Symp. 1999 Antennas and Propagation Society, vol. 2, Orlando, FL, 1999, pp. 1388-1391.

[9] - "On chaotic electromagnetic wave propagation," Microwave Opt. Technol. Lett., vol. 21, no. 6, pp. 448-451, 1999.

[10] R. L. Haupt and S. E. Haupt, Practical Genetic Algorithms. New York: Wiley, 1998.

[11] H. Chou, G. Premkumar, and C. Chu, "Genetic algorithm for communications network design- an empirical study of the factors that influence performance," IEEE Trans. Evolutionary Computation, vol. 5, pp. 236-249, June 2001.

[12] H. Sayoud, K. Takahashi, and B. Vaillant, "Designing communication network topologies using steady-state genetic algorithms," IEEE Trans. Commun., vol. 5, pp. 113-115, Mar. 2001.

[13] C. Ergun and K. Hacioglu, "Multiuser detection using a genetic algorithm in CDMA communications systems," IEEE Trans. Evolut. Computat., vol. 48, pp. 1374-1383, Aug. 2000.

[14] Z. Altman, R. Mittra, and A. Boag, "New designs of ultra wide-band communication antennas using a genetic algorithm," IEEE Trans. Antennas Propagat., vol. 45, pp. 1494-1501, Oct. 1997.

[15] T. C. Fogarty and L. Bull, "Optimizing individual control rules and multiple communicating rule-based control systems with parallel distributed genetic algorithms," Proc. IEEE—Contr. Theory Applicat., vol. 142, pp. 211-215, May 1995.

[16] T. L. Carroll and L. M. Pecora, "Synchronizing chaotic circuits," IEEE Trans Circuits Syst., vol. 38, pp. 453-456, Apr. 1991. 